\title{
Magnetic Carbon Composite Particles for Dye Adsorption from Water and their Electrochemical Regeneration
}

Michael Schneider, Thomas Ballweg, Lennart Groß, Carsten Gellermann, Angela SánchezSánchez, Vanessa Fierro, Alain Celzard, Karl Mandel*

M. Schneider, T. Ballweg, L. Groß, Dr. C. Gellermann, Dr. K. Mandel

Fraunhofer Institute for Silicate Research, ISC

Neunerplatz 2

97082 Wuerzburg, Germany.

Dr. K. Mandel

Chair of Chemical Technology of Materials Synthesis, University Wuerzburg

Roentgenring 11

97070 Wuerzburg, Germany

E-Mail: karl-sebastian.mandel@isc.fraunhofer.de

Dr. A. Sánchez-Sánchez, Dr. V. Fierro, Prof. A. Celzard

Institut Jean Lamour, UMR CNRS -Université de Lorraine 7198, ENSTIB

27 rue Ph. Seguin, BP 21042

88051 Epinal Cedex 9, France

Keywords: magnetic particles, carbon coating, electrochemical regeneration, dye adsorption, water purification

Abstract

Herein, the development of a novel composite particle system prepared from superparamagnetic $\mathrm{Fe}_{3} \mathrm{O}_{4} @ \mathrm{SiO}_{2}$ microparticles as core and coated with a carbon shell is presented. The coating with carbon was done by pyrolyzing furan resin which was previously deposited on the particle surface by polymerization of furfuryl alcohol. The novel composite material thus combines magnetic and sorptive properties. Upon pyrolysis the magnetic material was converted from magnetite to $\alpha$-iron and fayalite, changing its behavior from superparamagnetic to ferromagnetic with nearly zero remanence. The magnetic properties can be utilized to magnetically collect the particles dispersed in fluids, whereas the sorptive properties of the carbon shell can be used to remove organic contaminants from these fluids. The adsorption behavior of the composite particles and a potential electrochemical regeneration route were investigated, using the model dye methylene blue. 


\section{Introduction}

Impurities found in water are manifold nowadays and include in particular a huge variety of organic molecules such as dyes, ${ }^{[1-5]}$ pesticides, ${ }^{[6-8]}$ hormones, drugs ${ }^{[9-11]}$ and many more. It is one of today's urgent challenges to find efficient ways to remove such organic trace substances from (waste) water. An energy-efficient way is using adsorbent materials for this task, and among these a promising class is carbon-based materials. Due to their $\pi$-electrons and their high surface area, carbon-based materials are capable of adsorbing a lot of unwanted organic substances from water ${ }^{[12,13]}$. Yet, to be most efficient, the adsorbent material should ideally be finely distributed in the water body as this would mean short diffusion paths for the target substances towards the active adsorbent surface. Thus, by dispersing the adsorbent in the form of small particles, myriads of active adsorbent sites are instantly available to interact with the target contaminants in the water, making the adsorption process highly efficient and fast. However, the challenge is then to separate the small, organic-loaded adsorbents from water. An attractive solution might be the use of particles having magnetic properties, since collecting them in a magnetic field gradient would be possible. Ideally, such particles should present superparamagnetic behavior which means that they would act like switchable magnets due to a special nano-property (which is discussed in great detail elsewhere ${ }^{[14]}$ ). In fact, recent work demonstrated the successful combination of carbons with magnetic nanoparticles to generate a particle system which acts as magnetically recoverable adsorbent. Examples are magnetic carbon nanotubes, ${ }^{[15-20]}$ magnetic graphene, ${ }^{[21-24]}$ as well as magnetic activated carbon $^{[25-29]}$. Typically the carbon material is synthesized prior to the functionalization with magnetic particles. As magnetic material, magnetite $\left(\mathrm{Fe}_{3} \mathrm{O}_{4}\right)$ is predominantly used, which is either mixed with the carbon material in the form of nanoparticles, ${ }^{[16-18]}$ or is synthesized in situ from iron salts in the presence of the carbon material. ${ }^{[15,19-21,23-29]}$

Most reported works have in common that the magnetic and the carbon building blocks are just connected by light interaction forces or by being geometrically entangled. Yet, concerns 
may arise regarding the long-term stability of such rather lose and presumably mechanically not very stable formations. For this reason, we herein planned to develop micron-sized particles containing magnetic nanoparticles, safely enclosed in a silica matrix which was finally coated with a carbon layer. The latter was obtained by deposition and polymerization of furfuryl alcohol at the silica surface and subsequent pyrolysis.

Besides ensuring a stable combination of building blocks that renders magnetic separability and good adsorption of organics possible at the same time, another challenge is the regeneration of the magnetic carbon particles after adsorption and magnetic separation. Chemical regeneration attempts were reported, either by changing the $\mathrm{pH}^{[30-32]}$ or by adding chemicals such as solvents ${ }^{[33,34]}$ to regenerate carbon-based particles. Thinking in terms of a sustainable process applicable on a larger (real life) scale, the use of chemicals, in particularly solvents, for regenerating adsorbent particles is highly unwanted. Regeneration by furnace processes to burn the adsorbed organics, as often done for (macroscopic) carbons, ${ }^{[31,32,35,36]}$ is not ideal either, because firstly, it is energy-consuming and secondly, in case of magnetic particles containing carbon, oxidation of the magnetic components and thus loss of magnetic properties might be faced. For this reason, we studied alternative regeneration processes for the particles developed in this work and found indications that it is possible to exploit electrochemical principles to recover a part of the particles' adsorption capability. This proof of concept demonstration might open up a potentially promising avenue towards low energydemanding, chemical-free regeneration of carbon adsorbents, which, in combination with magnetic recovery, is a highly innovative new approach. 


\section{Results and Discussion}

\subsection{Synthesis and characterization of the magnetic carbon $\mathrm{Fe}-\mathrm{X} @ \mathrm{SiO}_{2} @ \mathrm{C}$ composite particle system}

Micron-sized magnetic microparticles, herein referred to as $\mathrm{Fe}-\mathrm{X} @ \mathrm{SiO}_{2} @ \mathrm{C}$ and denoted as “carbon-coated particles", were obtained by firstly incorporating superparamagnetic iron oxide nanoparticles in a silica matrix, made from water glass, as reported before ${ }^{[37]}$ followed by carbonization of the micron-sized $\mathrm{Fe}_{3} \mathrm{O}_{4} @ \mathrm{SiO}_{2}$ composite particles prepared as follows. The $\mathrm{Fe}_{3} \mathrm{O}_{4} @ \mathrm{SiO}_{2}$ composite particles, denoted as "carrier particles", were stirred in a solution of furfuryl alcohol which was subsequently polymerized at the silica surface by adding acid to the alcohol solution containing the particles. By optimization of the suitable ratios of the constituents, it was possible to generate a polymer shell around the carrier particles. Subsequently, the particles were magnetically extracted from the alcohol solution and placed in a furnace to undergo pyrolysis under nitrogen atmosphere at $900{ }^{\circ} \mathrm{C}$ for $2 \mathrm{~h}$ (Scheme 1). The term "Fe-X" was chosen for highlighting that such pyrolysis could induce reactions between $\mathrm{Fe}_{3} \mathrm{O}_{4}$ and carbon on the one hand, and between $\mathrm{Fe}_{3} \mathrm{O}_{4}$ and $\mathrm{SiO}_{2}$ on the other hand (details see below).

After heat-treatment, the particles were found to be agglomerated / aggregated. However, grinding for 5 minutes by hand (mortar and pestle) was sufficient to break up the product into individual particles which were found to resemble closely to the original carrier particles before carbonization (Figure 1a,b). Fraunhofer diffraction on both carrier particles and carbon-coated particles redispersed in isopropyl alcohol showed a broader size distribution for the pyrolyzed carbon-coated particles with a slightly smaller mean diameter of about $10 \mu \mathrm{m}$ compared to $20 \mu \mathrm{m}$ for the carrier particles (Figure 1c).

X-ray diffraction (XRD) under conventional conditions revealed that the carbonization obviously caused a change in the nanoparticles crystal structure and composition. The typical magnetite / maghemite diffraction reflections of the iron oxide nanoparticles (tagged with 
“*”) vanished and instead, reflections which could be attributed to $\alpha$-iron (tagged with "»") and to fayalite $\left(\mathrm{Fe}_{2} \mathrm{SiO}_{4}\right.$, tagged with "o") were observed (see Figure 1d). According to the XRD measurements, the crystalline part of the material consists of about $15 \%$ of $\alpha$-iron and $85 \%$ of fayalite. Thus, it is apparent that the carbon produced by the thermal decomposition of (poly)furfuryl alcohol reacted with iron oxide nanoparticles, partly reducing them to pure iron and partly transforming them to fayalite, thus involving the silica matrix in the reaction. It should be noticed that, despite pure nano-sized iron is a pyrophoric substance, its encapsulation in silica and carbon led to remarkably stable particles since it was found that, even after three months, no noticeable changes could be observed.

The reaction amongst the iron oxide nanoparticles also caused a change in their magnetic properties. While the original $\mathrm{Fe}_{3} \mathrm{O}_{4} @ \mathrm{SiO}_{2}$ carrier particles reveal a magnetization curve that is typical of a superparamagnetic system (no remanence, "S-shaped" curve) with a saturation magnetization of $30 \mathrm{emu} \mathrm{g}^{-1}$, the $\mathrm{Fe}-\mathrm{X} @ \mathrm{SiO}_{2} @ \mathrm{C}$ particles (carbon-coated particles) possess a magnetization curve which is "Z-shaped" and also reveal the absence of a measurable hysteresis (see Figure $\mathbf{2 b}$ around the point of zero magnetic field). Interestingly, the saturation magnetization of this system is higher than the one of the original carrier particles, namely $37 \mathrm{emu} \mathrm{g}^{-1}$, which means an increase by $23 \%$ in magnetization for this system. The magnetic hysteresis shape resembles that of a very soft, multi-domain system. Since the iron oxide was transformed to $\alpha$-iron and fayalite, both phases have an impact on the magnetization curve of the composite material. The Z-shape originates from the ferromagnetic $\alpha$-iron. As can be seen in Figure 2a, pure $\alpha$-iron also shows a Z-shaped magnetization curve, but with a significantly higher saturation magnetization. Fayalite is a paramagnetic material, therefore the magnetization curve is a straight line with a positive slope. Taking this into account, the magnetization curve of the sample material should be a mixture of the two properties. As can be seen in Figure 2c, the slope of the curve of pure $\alpha-$ iron is nearly zero at high magnetic fields. The curve of the carbon-coated particles, however, 
still reveals an increase in magnetization at high magnetic fields, indicating an additional paramagnetic contribution to the magnetic character of the system, which thus can be assigned to fayalite.

As a result, the soft magnetic properties of the $\mathrm{Fe}-\mathrm{X} @ \mathrm{SiO}_{2} @ \mathrm{C}$ particles still possess a nearly switchable magnetic behavior, i.e., in presence of a magnetic field gradient, the particles can be easily and fairly quickly collected, while they easily redisperse to single particles again when the magnetic field is removed.

To better understand the reactions occurring in the complex composite particles during pyrolysis, DSC, TG and temperature-dependent XRD were carried out. DSC and TG measurements were performed under either argon or synthetic air with a heating rate of $10{ }^{\circ} \mathrm{C} \min ^{-1}$. The results are shown in Figure 1e. As can be seen from the results under argon, no significant change in sample mass and no significant heat flow could be detected, which confirms that the conversion of the furan polymer into carbon was complete. The measurements under air show that there is a significant weight loss between 500 and $650{ }^{\circ} \mathrm{C}$ accompanied by a respective exothermic peak in DSC. This can be correlated to the burning / oxidation of the carbon coating. The weight loss is about $12 \%$, which indicates that the total amount of carbon in the composite particle should be roughly around $10 \%$.

Temperature-dependent XRD measurements show the transition of the magnetic material. A selection of patterns is shown in Figure 3, whereas the complete set of patterns can be seen in Figure $\mathbf{S 1}$ of the supplementary information. As can be seen, the significant reflections for magnetite marked with "*” occur up to a temperature of $600{ }^{\circ} \mathrm{C}$ and then disappear while significant reflections for fayalite (marked with "o") first appear at a temperature of $700{ }^{\circ} \mathrm{C}$. The two reflections marked with "王" occur in all patterns, irrespective to the temperature. These reflections can be attributed to the platinum heating plate used in the sample chamber. These findings confirm the direct transition from magnetite to fayalite, without the occurrence of an intermediate phase. The patterns up to $800{ }^{\circ} \mathrm{C}$ show a halo, typical for amorphous 
material such as silica which is part of the carrier particles. For a better comparison of the patterns, this halo was removed upon analysis and is not shown in Figure 3. The same hold true for Figure 1d. Nevertheless the disappearance of the amorphous halo is additional evidence that the amorphous silica-matrix is transformed into a crystalline material (presumably fayalite). Therefore the corresponding part of the patterns showing the decrease of the amorphous halo is shown in Figure $\mathbf{S 2}$ of the supplementary information, which, as it continuously disappears upon heating, gives additional evidence for the aforementioned transformation.

The texture of the carbon coating was investigated more closely by means of Raman spectroscopy. It was first observed that, wherever the laser beam was positioned on the material surface, strictly identical spectra typical of highly disordered carbon were always obtained (see Figure S3a of the Supplementary Information). This finding suggests that the carbon layer is homogeneous and completely coats the carrier particles, since no other bands but those of carbon could be seen. It also demonstrates that, as expected, the pyrolysis of polymerized furfuryl alcohol produced a non-graphitic carbon, as evidenced from several facts:

(i) a $1^{\text {st }}$-order part, ranging from 1000 to $1800 \mathrm{~cm}^{-1}$ and presenting an intense and broad D band (around $1345 \mathrm{~cm}^{-1}$ ) and a slightly narrower $\mathrm{G}$ band (around $1590 \mathrm{~cm}^{-1}$ ) of rather similar intensity, can be observed. However, the spectrum envelope can be very accurately deconvoluted into 4 mixed Gaussian-Lorentzian profiles usually called $\mathrm{D}_{4}, \mathrm{D}_{1}, \mathrm{D}_{3}$ and $\mathrm{G}$ in the literature, appearing at increasing wavenumbers ${ }^{[38]}$ (see Figure S3b). It is thus found that the $\mathrm{D}_{1} / \mathrm{G}$ intensity ratio is 1.32 , i.e., is much higher than 1 ;

(ii) a shallow valley between $\mathrm{D}_{1}$ and $\mathrm{G}$ bands, assigned to the contribution called $\mathrm{D}_{3}$, always present as a very broad but intense band in poorly crystallised carbons and attributed to defects outside the plane of aromatic layers such as tetrahedral carbons; ${ }^{[39,40]}$ 
(iii) a shoulder around $1200 \mathrm{~cm}^{-1}$, assigned to the very broad $\mathrm{D}_{4}$ band which is characteristic of very poorly organized carbons; ${ }^{[41]}$

(iv) and finally a $2^{\text {nd }}$-order part between 2200 and $3400 \mathrm{~cm}^{-1}$, whose lack of structuration into well-separated peaks near $2700 \mathrm{~cm}^{-1}$ proves the absence of tri-periodic order, i.e., which would have involved an order in the way the carbon layers are stacked. ${ }^{[42]}$

Decreasing the laser energy, $E_{L}$, from 2.33 to 1.95 and $1.58 \mathrm{eV}$ by changing the wavelength of the laser from 532 to 638 and $785 \mathrm{~nm}$, respectively, produced the expected phenomena ${ }^{[40]}$ shown in Figure S3c, i.e., a shift of the $D_{1}$ band to lower wavenumbers whereas the $G$ band remained at the same position but with a decreased intensity. The corresponding deconvolutions of the spectra obtained at 638 and $785 \mathrm{~nm}$ into 4 Gaussian-Lorentzian profiles are shown in Figures S3d and S3e, respectively.

Although only three incident energies were available for establishing quantitative trends, the intensity ratio of $\mathrm{D}_{1}$ to $\mathrm{G}$ bands was found to vary as $I_{D 1} / I_{G} \sim 1 / E_{L}$, and the $\mathrm{D}$ band was seen to shift with the increasing excitation energy at a rate of $68 \mathrm{~cm}^{-1} \mathrm{eV}^{-1}$. The way the $\mathrm{D}_{1} / \mathrm{G}$ intensity ratio and the shift of the $\mathrm{D}_{1}$ band change with laser incident energy, as well as the positions (shown in Figures S3b, S3d and S3e) and FWHM of $\mathrm{D}_{1}$ and $\mathrm{G}$ bands measured at $532 \mathrm{~nm}$ (around 155 and $70 \mathrm{~cm}^{-1}$, respectively), are consistent with each other and are typical of cokes having crystallite size typically less than $5 \mathrm{~nm} \cdot{ }^{[43]}$ These findings are plausible since the present materials were prepared from a non-graphitizable precursor pyrolyzed at a temperature not higher than $900^{\circ} \mathrm{C} .^{[44]}$

Figure 4 shows the nitrogen adsorption-desorption isotherm at $-196^{\circ} \mathrm{C}$, which is a combination of types $\mathrm{Ib}$ and $\mathrm{IV},{ }^{[45]}$ typical of micro-mesoporous materials. A horizontal plateau at $P / P_{0}>0.1$ was not reached due to multilayer adsorption in wide micropores and narrow mesopores, suggesting a continuous PSD. A hysteresis loop type $\mathrm{H} 2(\mathrm{a})^{[45]}$ closing at $P / P_{0}=0.43$ was observed together with a step desorption at $P / P_{0}=0.5$ that could be related to 
pore blocking-induced evaporation and represent a percolation transition. In this case, the confined liquid evaporates from the pore system when the liquid from the largest neck evaporates. Hysteresis loop type $\mathrm{H} 2$ (a) is also observed in the case where the size distribution of pore cavities is relatively wide compared to the distribution of neck sizes. Further argon adsorption-desorption at $87 \mathrm{~K}$ and scanning of the hysteresis cycle would be necessary to get more insight on the desorption process and pore structure. ${ }^{[46]}$

The $A_{B E T}$ was $164 \mathrm{~m}^{2} / \mathrm{g}$, somewhat lower than the $S_{N L D F T}$ of $176 \mathrm{~m}^{2} / \mathrm{g}$. It is well-known that the BET method underestimates the surface when only a monolayer can be adsorbed in narrow micropores while it overestimates the surface when pore filling as well as adsorption exists in wide micropores. Since $A_{B E T}$ is more commonly given in the literature, we present both values in Table 1.

Figure 5 a shows the PSD obtained by application of the 2D-NLDFT HS method considering slit-shaped pores in carbon materials. It also shows the cumulative volume that allows observing more easily the contribution of the micropores $(w<2 \mathrm{~nm})$ and mesopores $(2<w<$ $50 \mathrm{~nm}$ ) to the total pore volume. There is a bimodal PSD in the mesopore range: between 2 and $12 \mathrm{~nm}$ on the one hand, and between 18 and $29 \mathrm{~nm}$ on the other hand. Figure 5 b shows the relevance of the fit in the whole pore range. Finally, Table 1 shows the pore volume corresponding to each range of pore diameters. Mesopore volume, $V_{m e s}$, accounted for more than the half, $54 \%$, of the total pore volume, $V_{t o t}$, and the volume of pores narrower than 0.7 nm was particularly important, $29 \%$ of $V_{\text {tot }}$.

These analyses thus reveal that the carbon-coated particles (Fe-X @ $\left.\mathrm{SiO}_{2} @ \mathrm{C}\right)$ have a specific surface area of about $176 \mathrm{~m}^{2} \mathrm{~g}^{-1}$. This does not appear to be very high at first glance compared to other carbon systems. However, taking into account that the core particle is $\mathrm{Fe}-\mathrm{X}$ @ $\mathrm{SiO}_{2}$, assuming the weight content of this particle to be about $90 \%$, which can roughly be estimated through the DSC-TG measurements, there are two different ways to determine the specific surface area attributed to the carbon shell. 
Assuming that the particles are completely coated with carbon, so that only the carbon shell contributes to the specific surface area of the carbon-coated particles, the maximum value $x$ of the specific surface area of the carbon shell can roughly be estimated according to the following equation (1)

$0.1 \cdot \mathrm{x}=176 \mathrm{~m}^{2} \mathrm{~g}^{-1}$

Calculating $\mathrm{x}$ reveals that the maximum value of the specific surface area of the carbon component is $\approx 1760 \mathrm{~m}^{2} \mathrm{~g}^{-1}$. The minimum value $y$ of the specific surface area of the carbon shell can roughly be estimated, knowing that the carrier particles originally had a specific surface area of about $72 \mathrm{~m}^{2} \mathrm{~g}^{-1},{ }^{[37]}$ by using the following equation (2)

$0.9 \cdot 72 \mathrm{~m}^{2} \mathrm{~g}^{-1}+0.1 \cdot \mathrm{y}=176 \mathrm{~m}^{2} \mathrm{~g}^{-1}$

Calculating $y$ reveals that the minimum value of the specific surface area of the carbon component is $>1100 \mathrm{~m}^{2} \mathrm{~g}^{-1}$. These limit values are consistent with reported values of carbons prepared with furfuryl alcohol as carbon source. ${ }^{[47]}$ This indicates that the carbon shell obtained with the polymerization and pyrolysis process of furfuryl alcohol is an approach to create carbon materials with a remarkably high surface area.

2.2 Adsorption properties and electrochemical desorption with the magnetic carbon FeX@SiO2 @ C composite particle system

Having established a core-shell carbon magnetic particle composite system (carbon-coated particles), the adsorption properties of the system were investigated at $22{ }^{\circ} \mathrm{C}$. For this purpose, the model organic dye methylene blue was used, which is a common approach to evaluate the adsorption behavior of carbons towards organic pollutants in water. ${ }^{[1,5,48]}$ Because it is wellknown that $\mathrm{MB}$ is a cationic dye whose adsorption is highly sensitive to both $\mathrm{pH}$ and ionic strength, ${ }^{[49-53]}$ we decided to fix these parameters by working in the conditions detailed in the experimental section, using a mixture of deionized water and ethanol (ratio water : ethanol = 9 : 1) ( $\mathrm{pH} 7-8)$. Figure 6 depicts the adsorption kinetics and the adsorption isotherm of the carbon-coated particles. 
Figure 6a shows the adsorption kinetics for the methylene blue uptake. The purpose of this experiment was to estimate the time after which the equilibrium was reached, which is absolutely necessary before getting the adsorption isotherm shown in Figure 6b. After 70 min, $90 \%$ of the total dye uptake was achieved. The experimental data were fitted with both pseudo-first-order and pseudo-second-order kinetic models. Only the pseudo-second-order model showed a good compliance $\left(\mathrm{R}^{2}=0.999, k_{2}=0.00748 \mathrm{~g} \mathrm{mg}^{-1} \mathrm{~min}^{-1}, q_{e}=11.93 \mathrm{mg} \mathrm{g}^{-1}\right)$. In this model, the adsorption process, rather than the particle mass transfer process, is considered as the rate-limiting factor. ${ }^{[54]}$ Although this model is poorly informative in terms of mechanisms that actually occur at the adsorbent - adsorbate interface, it is very popular in the literature as it can very satisfactorily fit the adsorption kinetics of many adsorbents in the liquid phase (see for instance ${ }^{[55]}$ and refs therein). Moreover, the values obtained for both $k_{2}$ and $q_{e}$, around $7 \times 10^{-3} \mathrm{~g} \mathrm{mg}^{-1} \mathrm{~min}^{-1}$ and $12 \mathrm{mg} \mathrm{g}^{-1}$, respectively, are very typical of methylene blue adsorption on commercial activated carbon. ${ }^{[56]}$.

Eventually, also the adsorption isotherm at equilibrium conditions was acquired. It is shown in Figure 6b. The experimental data were analyzed with the common Langmuir and Freundlich models (also shown in Figure 6b) and the generated equation parameters are listed in Table 2.

The Langmuir model describes the isotherm data better (compare the $\mathrm{R}^{2}$ values in Table 1 ), which indicates a single layer coverage of the surface, and is valid for adsorption on homogeneous surfaces only. For porous carbons presenting a high surface heterogeneity, both geometrical (e.g., different pore sizes and shapes) and chemical (e.g., functional groups, impurities), Freundlich isotherm should better fit the experimental data. ${ }^{[57]}$ Given that Langmuir fits better the adsorption isotherms, the carbon shell can be assumed to be such that: (i) adsorption is localized; (ii) all the active sites on the surface have similar energies; (iii) no interaction between adsorbed molecules exists; and (iv) the limiting reaction step is the surface reaction, just like in a heterogeneous catalytic reaction. These assumptions are quite 
reasonable, considering that the carbon shell coating the magnetic microparticles is quite thin and prepared from one single carbon source: pure polyfurfuryl alcohol.

The maximum sorption capacity according to the Langmuir model fit is about $29 \mathrm{mg}$ of dye per gram of carbon-coated particles. It is worth mentioning here that, despite the high specific weight of such particles (around $4.6 \mathrm{~g} \mathrm{~cm}^{-3}$, calculated from the respective fractions of carbon, iron and fayalite) and the correspondingly low amount of deposited carbon, the values of adsorption capacity shown in Figure $\mathbf{6 b}$ and that of $q_{\max }$ reported in Table $\mathbf{2}$ compare quite well with those formerly obtained for commercial granular activated carbons specially designed for water treatment. ${ }^{[56]}$ The maximum monolayer adsorption of MB onto various magnetic adsorbents was reviewed by two recent papers. ${ }^{[51,58]}$ The values were found to range from $15.7 \mathrm{mg} \mathrm{g}^{-1}$ for magnetic carbon nanotube ${ }^{[59]}$ to more than $600 \mathrm{mg} \mathrm{g}^{-1}$ for magnetic NP@starch-g-poly(vinyl sulfate). ${ }^{[60]}$ Our own monolayer capacity, close to $30 \mathrm{mg} \mathrm{g}^{-1}$, thus appears to be in the bottom of the ranking, but it should be emphasized that very few magnetic materials having monolayer capacities above $100 \mathrm{mg} \mathrm{g}^{-1}$ have been reported and that most of them rather range from 20 to $40 \mathrm{mg} \mathrm{g}^{-1}$.

Regeneration of the carbon-coated particle system prepared in this work was attempted by using electrochemical principles. In case of a proper coating of the magnetic particles with carbon, the resultant system should be conductive and thus electrostatically chargeable. Consequently, a change of the electrostatic charge stored at the surface might influence the interaction of the adsorbed dyes with this surface. The idea was to provide a constant fluid stream running over the particles maintained in contact with an electrode. Any induced desorption effect of the dye should then allow the constant fluid stream to carry away the dye. Figure 7 shows a principle sketch and a photograph of the experimental setup.

As induced charges could potentially alter / change / decompose the methylene blue dye in a way that its optical behavior might also be altered, concentration measurements by means of calibrated UV-Vis absorption as method to judge the effect of the electric field-induced 
desorption were not made. Instead, the following experiment was conducted, to see if the electric field applied to the particles had an effect on the desorption behavior: Particles were loaded with dye at a concentration which was close to saturation (as deduced from the isotherm, Figure 6b). Subsequently, the particles were exposed to an electric field, in order to release the adsorbed dye molecules. The experiment was conducted for particles exposed to different electric fields. A successful regeneration was verified indirectly by exposing the particles to fresh dye solution after electrochemical treatment. The amount of adsorbed dye, when subsequently used again to remove dye from water, was evaluated by measuring the change in dye concentration in the water, wherein the particles acted as adsorbent after electrochemical treatment. This concentration change was determined by means of calibrated UV-Vis absorption measurements (see experimental section for details). Figure 8a depicts the adsorption capacity of the particles in a fresh dye solution after having been exposed to different electric fields tested for regenerating the formerly dye-loaded particles. A clear correlation between applied electric field and adsorbent activity can be seen. This clearly indicates that particles can be reactivated when submitted to an electrochemical treatment after a first adsorption event. It should be noted that this is a first proof of concept and that the underlying mechanisms could not be elucidated yet. Further studies are still needed to determine whether it is a real desorption of the dye, a decomposition, or any other mechanism. Moreover, it is obvious that, despite there is a clear and reproducible effect of the applied electric field, the regeneration potential of the particles is not yet very high. Thus, a deeper understanding, which might be subject to future work, could probably contribute to optimize the regeneration process. For instance, a longer time of treatment in the plate capacitor might increase the regeneration effect.

Yet, it could be demonstrated that the process is also repeatable in several cycles. Figure $\mathbf{8 b}$ depicts the respective adsorption capacity after each regeneration step for three consecutive adsorption-regeneration-cycles while applying an electric field of either $80 \mathrm{~V} \mathrm{~m}^{-1}$ or 
$400 \mathrm{~V} \mathrm{~m}^{-1}$, corresponding to an electrical potential of $2 \mathrm{~V}$ or $10 \mathrm{~V}$, respectively, using the same particles in all three cycles. The regained adsorption capacity is roughly constant over the three cycles. Since the experimental setup is very simple the differences between the different cycles can be attributed to intrinsic errors. Nevertheless, and just like in Figure 8a, the correlation between applied electric field and regained adsorption capacity can also be seen in these experiments.

\section{Conclusion}

We herein developed a composite microparticle system combining good magnetic and sorptive properties. It was prepared from $\mathrm{Fe}_{3} \mathrm{O}_{4} @ \mathrm{SiO}_{2}$ superparamagnetic core particles which were coated with a carbon shell synthesized in situ by pyrolysis of a furan resin on the particle surface. Upon pyrolysis the magnetic component was altered from magnetite to $\alpha$-iron and fayalite, resulting in ferromagnetic behavior with a very low magnetic remanence, close to zero. This particle system is suitable for the adsorption of organic contaminants from aqueous solutions, which could be evidenced for the adsorption and magnetic extraction of the model organic dye methylene blue from water. An electrochemical approach for the regeneration of the dye-loaded particles was investigated. Depending on the applied electric potential, a partial regeneration of the adsorption capacity could be achieved, which is a first demonstration for such approach towards a cyclic use of adsorbent particles.

\section{Experimental Section}

Reagents and Materials: Iron(III) chloride hexahydrate $\left(\mathrm{FeCl}_{3} \cdot 6 \mathrm{H}_{2} \mathrm{O}, 99 \%+\right)$, iron(II) chloride tetrahydrate $\left(\mathrm{FeCl}_{2} \cdot 4 \mathrm{H}_{2} \mathrm{O}, 99 \%+\right)$ and furfuryl alcohol $\left(\mathrm{C}_{5} \mathrm{H}_{6} \mathrm{O}_{2}, 98 \%\right)$ were purchased from Sigma Aldrich. Ammonium hydroxide solution (aqueous $\mathrm{NH}_{3}, 25 \mathrm{wt} \%$ ), nitric acid $\left(\mathrm{HNO}_{3}\right.$, $1 \mathrm{M}$, diluted from a $53 \mathrm{wt} \%$ solution) and sodium silicate (water glass) solution (36 wt $\%$, molar ratio of $\mathrm{SiO}_{2}: \mathrm{Na}_{2} \mathrm{O}=3: 1, \mathrm{Na}_{2} \mathrm{Si}_{3} \mathrm{O}_{7}$ ) were purchased from Fishar Chemicals. 
Hydrochloric acid ( $\mathrm{HCl}, 1 \mathrm{M}$, diluted from a $37 \mathrm{wt} \%$ solution) was purchased from Carl Roth. All chemicals were used without further purification.

Synthesis of Carbon-coated Magnetic Composite Particles: The synthesis of $\mathrm{Fe}_{3} \mathrm{O}_{4} @ \mathrm{SiO}_{2}$ magnetic particles is based on a procedure published elsewhere. ${ }^{[37]}$ For the formation of the carbon coating, $20 \mathrm{~g}$ of such magnetic particles were put into a $1 \mathrm{~L}$ round flask. A mixture of furfuryl alcohol $(100 \mathrm{~mL})$ and deionized water $(300 \mathrm{~mL})$ was added and the mixture was stirred magnetically for $1 \mathrm{~h}$. Afterwards the particles were separated with a handheld magnet, the supernatant was removed, hydrochloric acid $(1 \mathrm{M}, 800 \mathrm{~mL})$ was added, and the mixture was stirred magnetically for another $5 \mathrm{~h}$ for polymerizing the furfuryl alcohol into a furan resin. Subsequently, the particles were again magnetically separated and washed five times with deionized water. The separated solid was then kept at room temperature for $12 \mathrm{~h}$ and dried for $5 \mathrm{~h}$ at $120^{\circ} \mathrm{C}$. Thereafter the dried material was ground to fine powder and prepared in ceramic beakers for pyrolysis. The pyrolysis was performed under nitrogen atmosphere, with a heating rate of $1^{\circ} \mathrm{C} \min ^{-1}$, a maximum temperature of $900{ }^{\circ} \mathrm{C}$, and a dwell time at maximum temperature of $2 \mathrm{~h}$. Finally the product was ground to powder again.

Adsorption of Methylene Blue on Carbon-coated Particles: To determine the adsorption behavior of the carbon-coated magnetic particles, adsorption tests with methylene blue were carried out, by considering both adsorption kinetics and isotherms. All experiments were carried out at $22{ }^{\circ} \mathrm{C}$ and in a mixture of deionized water and ethanol (ratio water : ethanol $=9: 1)$.

Adsorption kinetics: For the determination of adsorption kinetics, experiments were carried out with an initial methylene blue concentration of $100 \mathrm{mg} \mathrm{L}^{-1}$. The amount of carbon-coated particles was set to $4 \mathrm{~g} \mathrm{~L}^{-1}$ and the adsorption time was varied stepwise from 10 to $240 \mathrm{~min}$. During adsorption the mixtures were constantly stirred. The solid-liquid separation was performed with a handheld magnet. The remaining amount of dissolved methylene blue was determined by UV/Vis spectroscopy. Doing so, the peak intensity in the corresponding 
absorption spectrum was determined and the residual concentration in water was calculated from a calibration curve. The experimental data were analyzed with pseudo-first-order (Equation (3)) and pseudo-second-order (Equation (4)) kinetic models. The respective equations can be expressed in a linear form as follows:

$\log \left(q_{e}-q_{t}\right)=\log q_{e}-\frac{k_{1}}{2.303} \cdot t$

$\frac{t}{q_{t}}=\frac{1}{k_{2} \cdot q_{e}{ }^{2}}+\frac{t}{q_{e}}$

where the rate constants for the pseudo-first-order and pseudo-second-order kinetic models are $k_{1}$ and $k_{2}$, respectively. The amounts of methylene blue adsorbed at time $t$ and at equilibrium are $q_{t}$ and $q_{e}$, respectively.

Adsorption isotherms: The experiments for the determination of the adsorption isotherms were carried out in a similar manner as the kinetics experiments. The concentration of carboncoated particles was kept constant at $4 \mathrm{~g} \mathrm{~L}^{-1}$ and the initial concentration of methylene blue was varied from 75 to $250 \mathrm{mg} \mathrm{L}^{-1}$. During adsorption, the suspensions were stirred at room temperature until equilibrium was reached. For sampling, the same processes as in the kinetics experiments were conducted. The experimental data were analyzed with Freundlich and Langmuir models. The equations of Freundlich (Equation (5)) and Langmuir (Equation (6)) can be expressed in the following linear form:

$\ln q_{e}=\ln K_{F}+n \cdot \ln C_{e}$

$\frac{C_{e}}{q_{e}}=\frac{1}{K_{L} \cdot q_{\max }}+\frac{1}{q_{\max }} \cdot C_{e}$

where the sorption coefficient for Freundlich and Langmuir models are $K_{F}$ and $K_{L}$, respectively. The methylene blue uptake capacity and the concentration of dissolved methylene blue at equilibrium are expressed as $q_{e}$ and $C_{e}$, respectively. The maximum adsorbed amount of methylene blue in a complete monolayer is $q_{\max }$, and $n$ is a constant.

Electrochemical Desorption/Degradation of Methylene Blue from Carbon-coated Particles: To investigate the electrochemically-induced desorption/degradation of methylene blue by the 
carbon-coated particles, two kinds of tests were performed by submitting them to various applied electric fields and measuring their sorption properties after several consecutive adsorption/regeneration cycles. Similar to the adsorption experiments, such tests were carried out in a mixture of deionized water and ethanol (ratio water : ethanol $=9: 1$ ), further denoted as "solvent".

Variation of electric field: The experiments to determine the influence of the electric field on the desorption/degradation of methylene blue from carbon-coated particles were carried out according to several consecutive steps. First $70 \mathrm{mg}$ of carbon-coated particles were mixed with $20 \mathrm{~mL}$ of dye solution with a concentration of $400 \mathrm{mg} \mathrm{L}^{-1}$ to obtain maximum loading of the particles. Then the particles were magnetically separated and washed one time with solvent to remove residual dye that was not adsorbed on the particle surface. Afterwards the loaded particles were transferred into a plate capacitor and hold in place by a handheld magnet. While applying the desired electric field $\left(0-1120 \mathrm{~V} \mathrm{~m}^{-1}\right)$, solvent was poured over the particles without creating a contact between the two plates of the capacitor design. After one minute of treatment the particles were removed and mixed with a solution of methylene blue with a concentration of $10 \mathrm{mg} \mathrm{L}^{-1}$ for $5 \mathrm{~min}$. After removing the particles magnetically one last time, the supernatant was analyzed by UV/Vis spectroscopy to determine the residual dye concentration as described before.

Use of particles in consecutive adsorption/regeneration cycles: The experiments were carried out in the same way as for the experiments described before. After loading the carbon-coated particles $\left(70 \mathrm{mg}\right.$ ) with dye (in a $400 \mathrm{mg} \mathrm{L}^{-1}$ solution), a total of 3 regeneration-adsorption cycles were conducted applying an electric field of either 80 or $400 \mathrm{~V} \mathrm{~m}^{-1}$, comprising the following steps: 1 . While applying the electric field, solvent was poured over the particles for one minute. 2. The particles were removed and mixed with a solution of methylene blue with a concentration of $10 \mathrm{mg} \mathrm{L}^{-1}$ for $5 \mathrm{~min}$. 3. The particles were removed magnetically and the supernatant stored for further analyses. 4. The particles were placed in the plate capacitor 
again. The supernatants of all cycles were analyzed by UV/Vis spectroscopy to determine the residual dye concentrations as described before.

Analytical Instrumentation: Investigations of the morphology of the material were performed with a scanning electron microscope (SEM) using a Zeiss Supra 25 SEM working at $3 \mathrm{keV}$ (field emission). X-ray diffraction (XRD) at constant temperature was performed on dried powder samples. A PANalytical 943006003002 Empyrean Series 2 X-ray diffractometer employing $\mathrm{Cu} \mathrm{K} \alpha$ radiation $(\lambda=0.15406 \mathrm{~nm}$ ) was used (step size $0.001652 \theta$, typical count time $60 \mathrm{~s}$ ). Temperature-dependent X-ray diffraction was performed on carrier particles coated with furan resin prior to pyrolysis. A PANalytical 943006003002 Empyrean Series 2 X-ray diffractometer employing Co $\mathrm{K} \alpha$ radiation $(\lambda=0.179 \mathrm{~nm})$ was used (step size $0.03942 \theta$, typical count time $100 \mathrm{~s}$ ) employing the sample chamber HTK 2000N from Anton Paar and a platinum heating plate. For a better comparison, the $2 \theta$ values of the XRD patterns acquired with the Co-source were transformed into $2 \theta$ values corresponding to $\mathrm{Cu} \mathrm{K} \alpha$ radiation. To simulate the conditions of pyrolysis, the sample was heated in a similar way as for the pyrolysis described before under nitrogen flow. While heating, XRD patterns were recorded while holding set temperatures and every 15 min during the dwell time at $900{ }^{\circ} \mathrm{C}$. The indexation of reflections was carried out referring to the International Centre for Diffraction Data PDF-4.

$\mathrm{N}_{2}$ adsorption studies were carried out using a Micromeritics 3Flex on degassed and dried samples $\left(80^{\circ} \mathrm{C}, 3 \cdot 10^{-2}\right.$ mbar, $\left.24 \mathrm{~h}\right)$. Microactive ${ }^{\circledR}$ software provided by Micromeritics was used to apply the BET method ${ }^{[61]}$ to $\mathrm{N}_{2}$ isotherm and obtain the BET area, $A_{B E T}$. Particular attention was paid to the $C$ parameter in the BET equation that provides information about the interaction between the adsorbent surface and the adsorbate, and which has to be positive. $A_{B E T}$ strongly depends on the range of relative pressures $\left(P / P_{0}\right)$ chosen to fit the BET equation to the nitrogen isotherm, and therefore it cannot be automatically applied between 0.05 and 0.25. We thus plotted $V_{N 2} \times\left(1-P / P_{0}\right)$, where $V_{N 2}$ is the adsorbed nitrogen volume at a given 
value of $P / P_{0}$, as a function of $P / P_{0}$ and starting at $P / P_{0}=0.01$. The maximum $P / P_{0}$ to fit the BET equation will be that where $P / P_{0} \times\left(1-P / P_{0}\right)$ reaches its maximum. ${ }^{[62]}$ The software SAIEUS®, also from Micromeritics, was used to apply the non-local density functional theory (NLDFT) to $\mathrm{N}_{2}$ adsorption isotherm. The 2D-NLDFT HS model ${ }^{[63]}$ was used to calculate the pore size distribution (PSD) and the following parameters: surface area, $S_{N L D F T}$; ultramicropore volume (corresponding to pore size $w<0.7 \mathrm{~nm}$ ), $V_{u \mu}$; supermicropore volume $(0.7<w<2 \mathrm{~nm}), V_{s \mu}$; micropore volume $(w<2 \mathrm{~nm}), V_{\mu}$; total pore volume, $V_{\text {tot }}$; and mesopore volume $(2<w<50 \mathrm{~nm}), V_{\text {meso }}=V_{\text {tot }}-V_{\mu}$.

Magnetic properties of the particles were recorded with a vibrating sample magnetometer (VSM, VersaLabTM 3T, Cryogen-free Vibrating Sample Magnetometer), cycling the applied field from -30 to $+30 \mathrm{kOe}$ two times with a step rate of $100 \mathrm{Oe} \mathrm{s}^{-1}$. Detailed analyses were carried out by cycling the applied field from -5 to $+5 \mathrm{kOe}$ at $5 \mathrm{Oe} \mathrm{s}^{-1}$. The temperature was set to $20^{\circ} \mathrm{C}$. Differential scanning calorimetry in combination with thermogravimetric analysis (DSC-TG) was carried out from room temperature to $900{ }^{\circ} \mathrm{C}$ and a heating rate of $10{ }^{\circ} \mathrm{C} \mathrm{min}{ }^{-1}$ with a Netzsch STA 449F3 by heating either in synthetic air (80 vol\% nitrogen, 20 vol\% oxygen) or in argon particles already pyrolyzed at $900{ }^{\circ} \mathrm{C}$. The $\mathrm{UV}-\mathrm{Vis}$ absorption spectra for the determination of dye concentration were measured in the range of $200-800 \mathrm{~nm}$ by a spectrometer Specord 50, Analytik Jena AG. The size distribution of particles in dispersion was determined by Fraunhofer diffraction (Microtrac S 350, Bluewave). To obtain good dispersions in isopropyl alcohol, the samples were treated with ultrasound for $5 \mathrm{~min}$ prior to analysis. Raman spectroscopy was carried out using an XploRa Raman spectrometer (Horiba) in the following conditions: microscope with a $50 \times$ objective, holographic grating with 1200 lines $\mathrm{mm}^{-1}$, accumulation of 2 spectra recorded from 200 to $3700 \mathrm{~cm}^{-1}$, lasers of wavelength 532, 638 or $786 \mathrm{~nm}$ filtered at $10 \%$ of their nominal power with acquisition times of 120,180 and 210 s, respectively. 


\section{Supporting Information}

Supporting Information is available from the Wiley Online Library or from the author.

\section{Conflicts of Interest}

The authors declare no conflict of interest.

\section{Acknowledgements}

This work was supported by the German Federal Ministry of Education and Research (BMBF) under the project ElektroWirbel (FKZ 13XP5008). The authors thank Jakob Reichstein and Hendrik Bohn for the support in the execution of the experiments in the lab.

Received: ((will be filled in by the editorial staff))

Revised: ((will be filled in by the editorial staff)) Published online: ((will be filled in by the editorial staff))

\section{References}

[1] V. K. Garg, M. Amita, R. Kumar, R. Gupta, Dyes Pigm., 2004, 63, 243.

[2] W. G. Kuo, Water Res., 1992, 26, 881.

[3] C.-C. Su, M. Pukdee-Asa, C. Ratanatamskul, M.-C. Lu, Desalination, 2011, 278, 211.

[4] H. Hayat, Q. Mahmood, A. Pervez, Z. A. Bhatti, S. A. Baig, Sep. Purif. Technol., 2015, $154,149$.

[5] P. Manoj Kumar Reddy, B. Rama Raju, J. Karuppiah, E. Linga Reddy, C. Subrahmanyam, Chem. Eng. J., 2013, 217, 41.

[6] N. Wardenier, P. Vanraes, A. Nikiforov, S. W. H. van Hulle, C. Leys, J. Hazard. Mater., 2019, 362, 238.

[7] L. Ghimici, I. A. Dinu, Sep. Purif. Technol., 2019, 209, 698.

[8] H. Bachmann Pinto, B. Miguel de Souza, M. Dezotti, J. Cleaner Prod., 2018, 201, 1061.

[9] H. Krause, B. Schweiger, E. Prinz, J. Kim, U. Steinfeld, J. Electrostatics, 2011, 69, 333.

[10] M. Magureanu, D. Piroi, N. B. Mandache, V. David, A. Medvedovici, V. I. Parvulescu, Water Res., 2010, 44, 3445.

[11] M. Magureanu, N. B. Mandache, V. I. Parvulescu, Water Res., 2015, 81, 124.

[12] S. J. T. Pollard, G. D. Fowler, C. J. Sollars, R. Perry, Sci. Total Environ., 1992, 116, 31.

[13] G. S. Simate, N. Maledi, A. Ochieng, S. Ndlovu, J. Zhang, L. F. Walubita, J. Environ. Chem. Eng., 2016, 4, 2291.

[14] M. Benz, Superparamagnetism - Theory and Applications-Discussion of Two Papers on Magnetic Nanoparticles, ETH, Zürich, 2012.

[15] G. Liu, L. Li, X. Huang, S. Zheng, X. Xu, Z. Liu, Y. Zhang, J. Wang, H. Lin, D. Xu, J. Mater. Sci., 2018, 53, 10772. 
[16] O. Duman, C. Özcan, T. Gürkan Polat, S. Tunç, Environ. Pollut., 2018, 244, 723.

[17] X. Fu, S. Liu, D. Zhu, Y. Xu, X. Yan, Mater. Lett., 2019, 234, 269.

[18] Y. Liu, Z. Zhao, D. Yuan, Y. Wang, Y. Dai, Y. Zhu, J. W. Chew, Appl. Surf. Sci., 2019, 466, 893.

[19] V. K. Gupta, S. Agarwal, T. A. Saleh, Water Res., 2011, 45, 2207.

[20] P. Wang, M. Cao, C. Wang, Y. Ao, J. Hou, J. Qian, Appl. Surf. Sci., 2014, 290, 116.

[21] K. Wu, C. Jing, J. Zhang, T. Liu, S. Yang, W. Wang, Appl. Surf. Sci., 2019, 466, 746.

[22] X. Wang, J. Lu, B. Cao, X. Liu, Z. Lin, C. Yang, R. Wu, X. Su, X. Wang, Colloid Surf. A-Physicochem. Eng. Asp., 2019, 560, 384.

[23] C. Wang, C. Feng, Y. Gao, X. Ma, Q. Wu, Z. Wang, Chem. Eng. J., 2011, 173, 92.

[24] P. Zong, S. Wang, Y. Zhao, H. Wang, H. Pan, C. He, Chem. Eng. J., 2013, 220, 45.

[25] W. Cai, Z. Li, J. Wei, Y. Liu, Chem. Eng. Res. Des., 2018, 140, 23.

[26] Z. Hao, C. Wang, Z. Yan, H. Jiang, H. Xu, Chemosphere, 2018, $211,962$.

[27] K. M. Lompe, S. Vo Duy, S. Peldszus, S. Sauvé, B. Barbeau, J. Hazard. Mater., 2018, $360,349$.

[28] D. Mohan, A. Sarswat, V. K. Singh, M. Alexandre-Franco, C. U. Pittman, Chem. Eng. $J ., 2011,172,1111$.

[29] N. Yang, S. Zhu, Di Zhang, S. Xu, Mater. Lett., 2008, 62, 645.

[30] Q. Li, Y. Qi, C. Gao, J. Cleaner Prod., 2015, 86, 424.

[31] E. Zhou, Y. He, X. Ma, G. Liu, Y. Huang, C. Chen, W. Wang, Chem. Eng. Process., 2017, 121, 224.

[32] S. W. Nahm, W. G. Shim, Y.-K. Park, S. C. Kim, Chem. Eng. J., 2012, 210, 500.

[33] D. Guo, Q. Shi, B. He, X. Yuan, J. Hazard. Mater., 2011, 186, 1788.

[34] M. A. Ferro-Garcia, J. Rivera-Utrilla, I. Bautista-Toledo, C. Moreno-Castilla, J. Chem. Tech. Biotechnol., 1996, 67, 183.

[35] D. Clifford, P. Chu, A. Lau, Water Res., 1983, 17, 1125.

[36] M. A. Waer, V. L. Snoeyink, K. L. Mallon, J. - Am. Water Works Assoc., 1992, 84, 82.

[37] K. Mandel, F. Hutter, C. Gellermann, G. Sextl, ACS Appl. Mater. Interfaces, 2012, 4, 5633.

[38] M. Pawlyta, J.-N. Rouzaud, S. Duber, Carbon, 2015, 84, 479.

[39] C. Bény-Bassez,J.-N. Rouzaud, Scanning Electron Microscopy, SEM Inc., 1985.

[40] A. Sadezky, H. Muckenhuber, H. Grothe, R. Niessner, U. Pöschl, Carbon, 2005, 43, 1731.

[41] O. Beyssac, B. Goffé, J.-P. Petitet, E. Froigneux, M. Moreau, J.-N. Rouzaud, Spectrochim. Acta, Part A, 2003, 59, 2267.

[42] P. Lespade, A. Marchand, M. Couzi, F. Cruege, Carbon, 1984, 22, 375.

[43] P. Mallet-Ladeira, P. Puech, C. Toulouse, M. Cazayous, N. Ratel-Ramond, P. Weisbecker, G. L. Vignoles, M. Monthioux, Carbon, 2014, 80, 629. 
[44] S. Bernard, O. Beyssac, K. Benzerara, N. Findling, G. Tzvetkov, G. E. Brown, Carbon, 2010, 48, 2506.

[45] M. Thommes, K. Kaneko, A. V. Neimark, J. P. Olivier, F. Rodriguez-Reinoso, J. Rouquerol, K. S.W. Sing, Pure Appl. Chem., 2015, 87, 1051.

[46] K. A. Cychosz, R. Guillet-Nicolas, J. García-Martínez, M. Thommes, Chem. Soc. Rev., 2017, 46, 389 .

[47] N. Li, J. Xu, H. Chen, X. Wang, J. Nanosci. Nanotechnol., 2014, 14, 5157.

[48] F. Huang, L. Chen, H. Wang, Z. Yan, Chem. Eng. J., 2010, 162, 250.

[49] Y. Song, Y. Duan, L. Zhou, J. Colloid Interface Sci., 2018, 529, 139.

[50] G. Gong, F. Zhang, Z. Cheng, L. Zhou, Int. J. Biol. Macromol., 2015, 81, 205.

[51] Z. Cheng, J. Liao, B. He, F. Zhang, F. Zhang, X. Huang, L. Zhou, ACS Sustainable Chem. Eng., 2015, 3, 1677.

[52] T. Selmi, M. Seffen, A. Celzard, V. Fierro, Environmental science and pollution research international, 2018, DOI: 10.1007/s11356-018-3835-8.

[53] Darmadi, Thomas S. Y. Choong, T. G. Chuah, Robiah Yunus, Y. H. Taufiq Yap, AJChE, 2008, 8, 27.

[54] Y.S. Ho, G. McKay, Process Biochem., 1999, 34, 451.

[55] V. Fierro, V. Torné-Fernández, D. Montané, A. Celzard, Microporous Mesoporous Mater., 2008, 111, 276.

[56] T. Selmi, M. Seffen, H. Sammouda, S. Mathieu, J. Jagiello, A. Celzard, V. Fierro, Adsorption, 2018, 24, 11.

[57] P. Girods, A. Dufour, V. Fierro, Y. Rogaume, C. Rogaume, A. Zoulalian, A. Celzard, J. Hazard. Mater., 2009, 166, 491.

[58] M. Oppmann, M. Wozar, J. Reichstein, K. Mandel, ChemNanoMat, 2018, 6, 141.

[59] J.-L. Gong, B. Wang, G.-M. Zeng, C.-P. Yang, C.-G. Niu, Q.-Y. Niu, W.-J. Zhou, Y. Liang, J. Hazard. Mater., 2009, 164, 1517.

[60] A. Pourjavadi, A. Abedin-Moghanaki, A. Tavakoli, RSC Adv., 2016, 6, 38042.

[61] S. Brunauer, P. H. Emmett, E. Teller, J. Am. Chem. Soc., 1938, 60, 309.

[62] F. Rouquerol, J. Rouquerol, K. Sing, Assessment of Surface Area, in: Adsorpt. Powders Porous solids, Academic Press, London, UK, 1999. pp. 165-189.

[63] J. Jagiello, J. P. Olivier, Adsorption, 2013, 19, 777. 


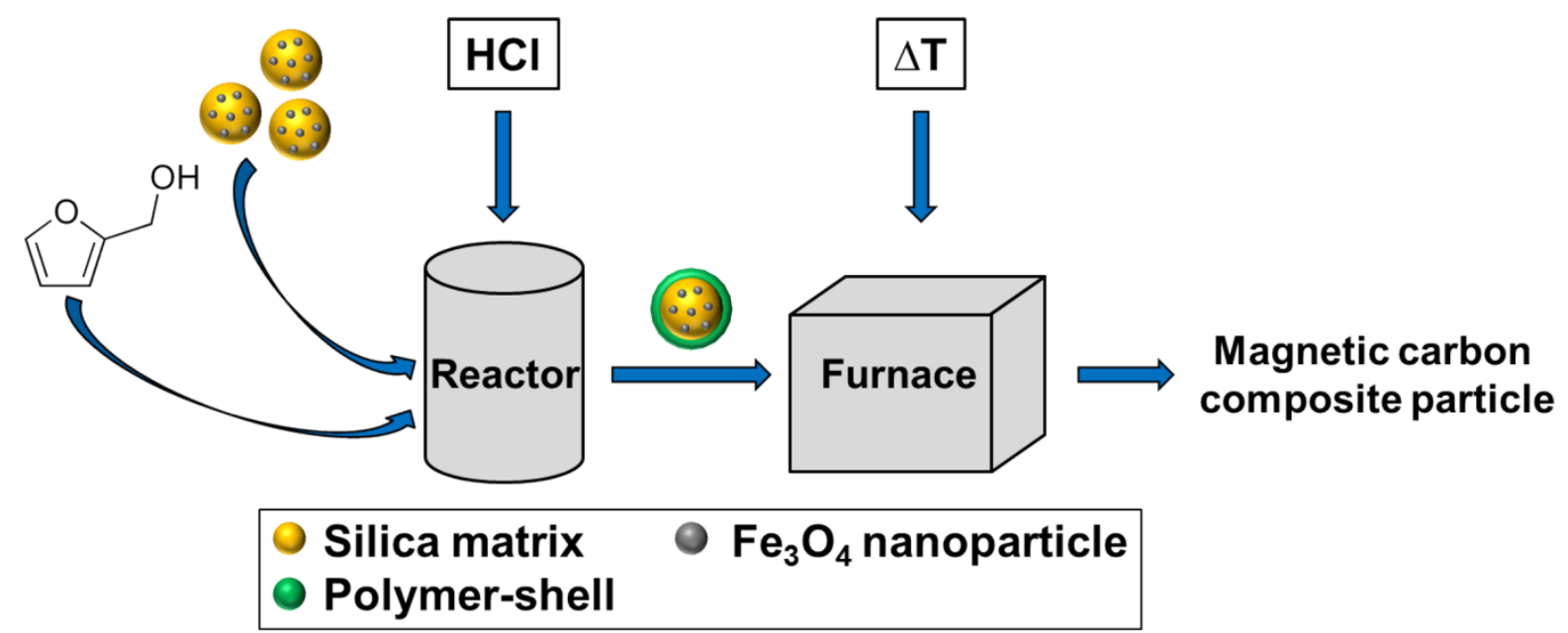

Scheme 1. Schematic depiction of the functionalization and transformation of $\mathrm{Fe}_{3} \mathrm{O}_{4} @ \mathrm{SiO}_{2}$ magnetic carrier particles with a carbon shell by coating with polymer and subsequent pyrolysis. 

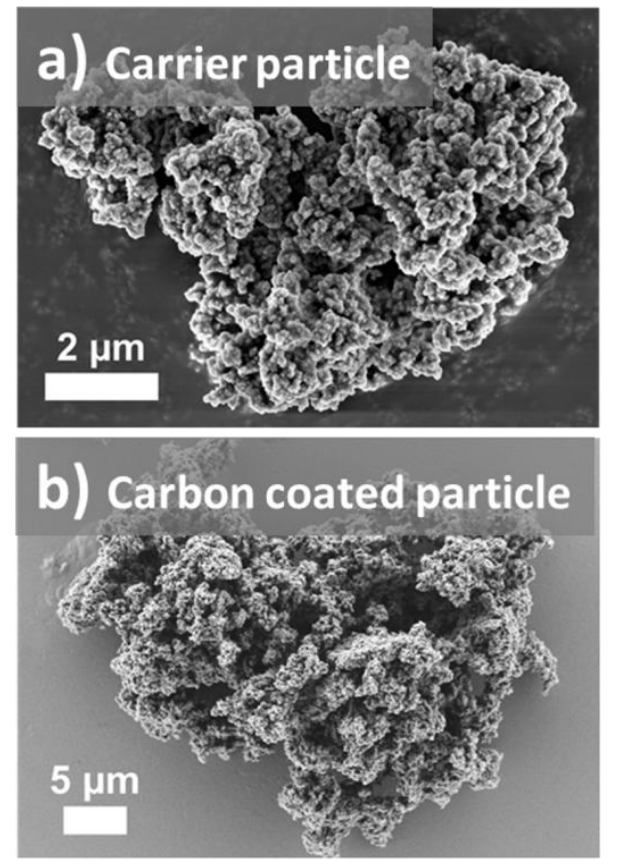

d)
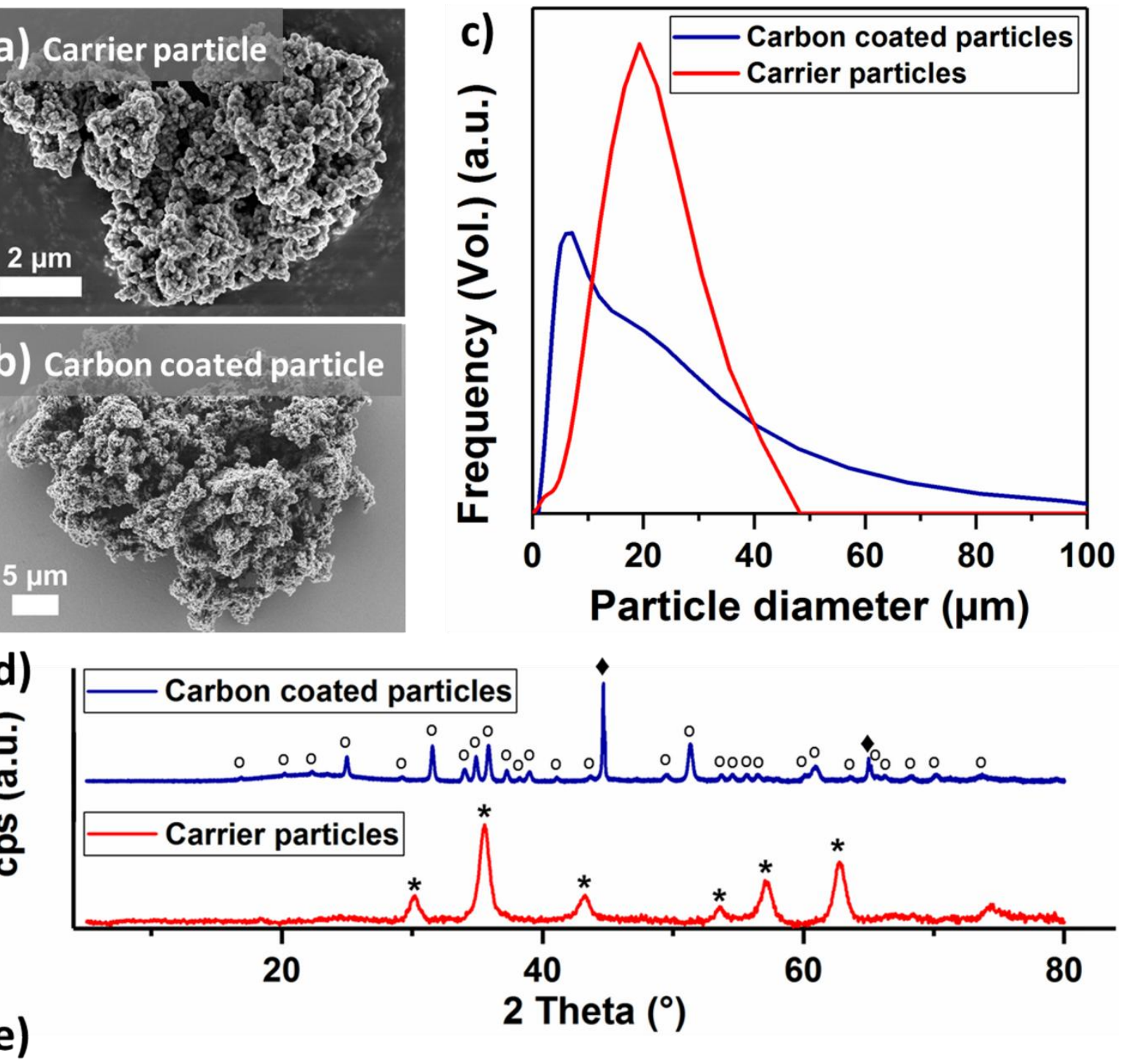

e)

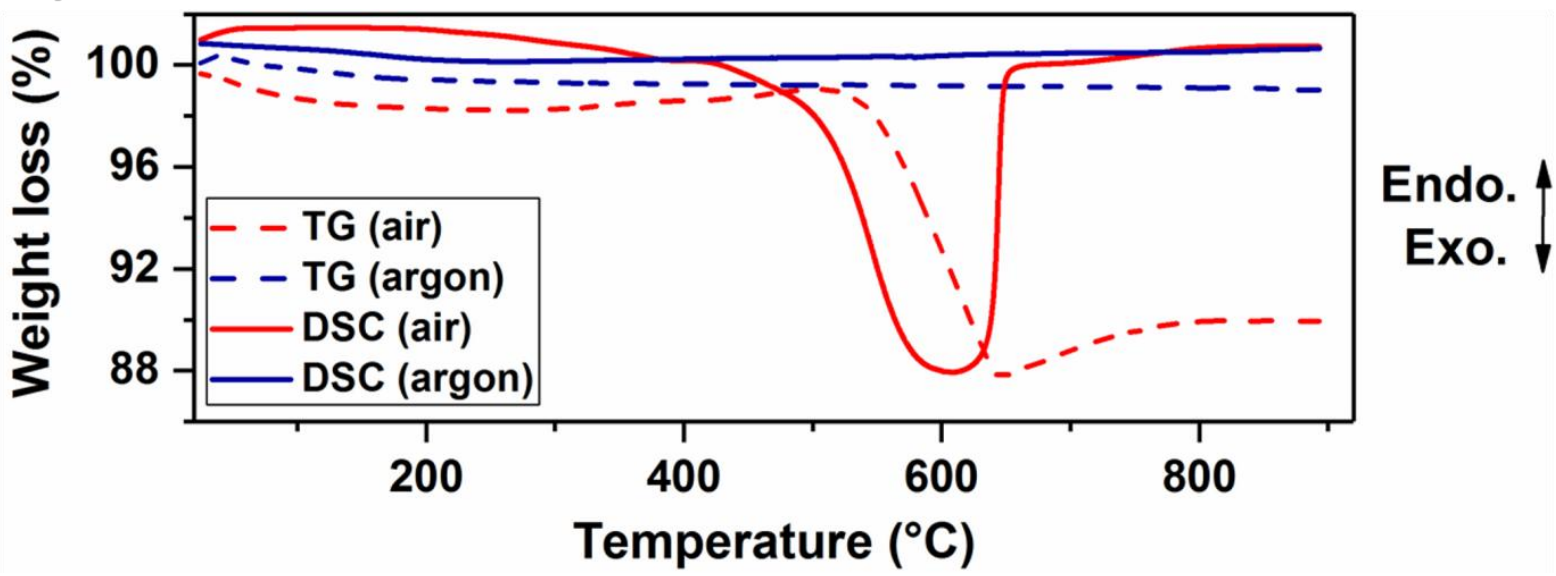

Figure 1. SEM-images of the carrier particles $\left(\mathrm{Fe}_{3} \mathrm{O}_{4} @ \mathrm{SiO}_{2}\right)$ (a) and the carbon-coated particles (Fe-X @ $\left.\left.\mathrm{SiO}_{2} @ \mathrm{C}\right)(\mathrm{b}) . \mathrm{c}\right)$ Fraunhofer diffraction of suspensions of carrier particles and carbon-coated particles in isopropyl alcohol. d) XRD patterns of carrier particles and carbon-coated particles: Magnetite / maghemite is indicated with "**", $\alpha$-iron is indicated with " " and fayalite is indicated with "o". e) DSC-TG of carbon-coated particles in synthetic air (80 vol\% nitrogen, 20 vol\% oxygen) and argon. 

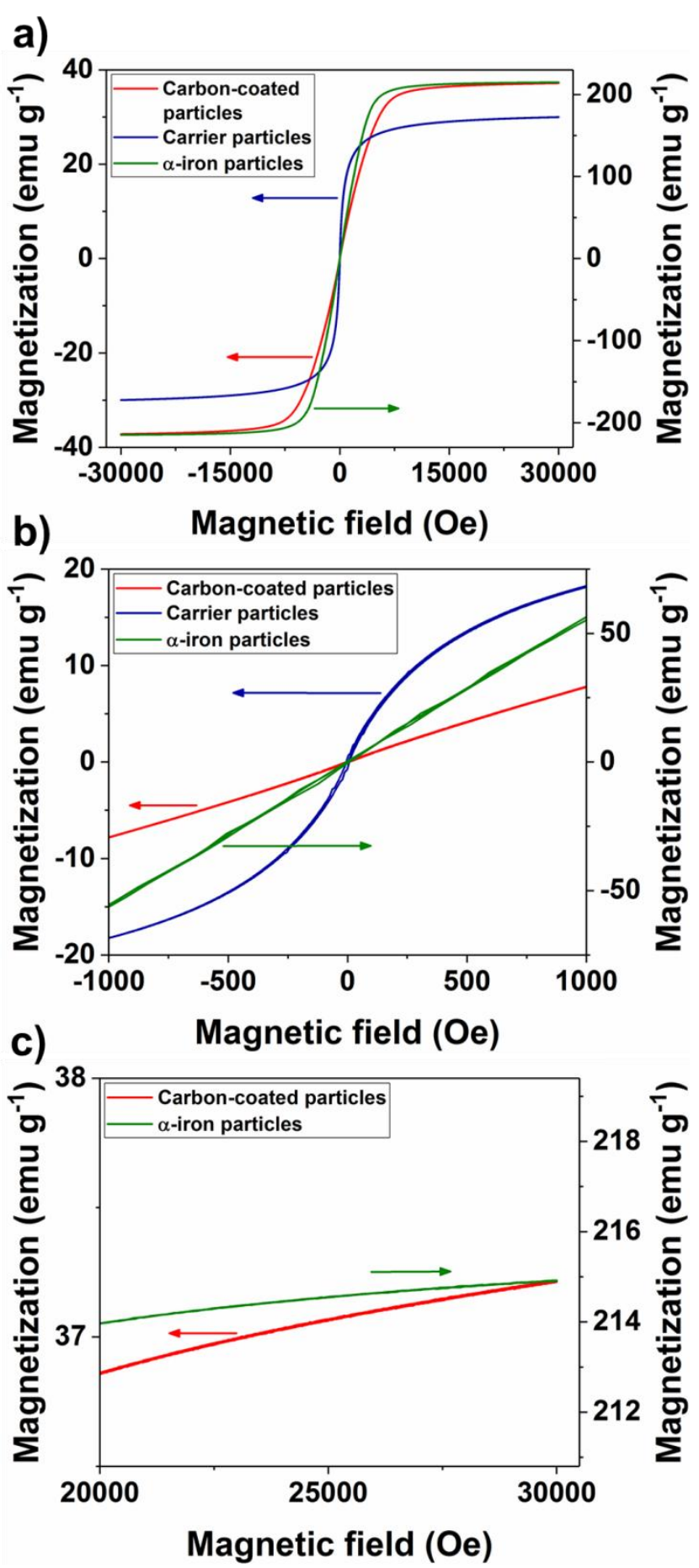

Figure 2. a) Magnetization curves of carbon-coated particles ( $\left.\mathrm{Fe}-\mathrm{X} @ \mathrm{SiO}_{2} @ \mathrm{C}\right)$ compared to the carrier particles $\left(\mathrm{Fe}_{3} \mathrm{O}_{4} @ \mathrm{SiO}_{2}\right)$ and $\alpha$-iron particles (commercial reference material from the company BASF). b) Detailed depiction of the magnetization curves at the point of zero external magnetic field. c) Detailed depiction of the magnetization curves at very high external magnetic fields for comparing carbon-coated particles and $\alpha$-iron particles. In all parts of the figure the magnetization curves of the carrier particles as well as the carboncoated particles refer to the y-axis on the left, whereas the magnetization curve of $\alpha$-iron particles refers to the y-axis on the right. 


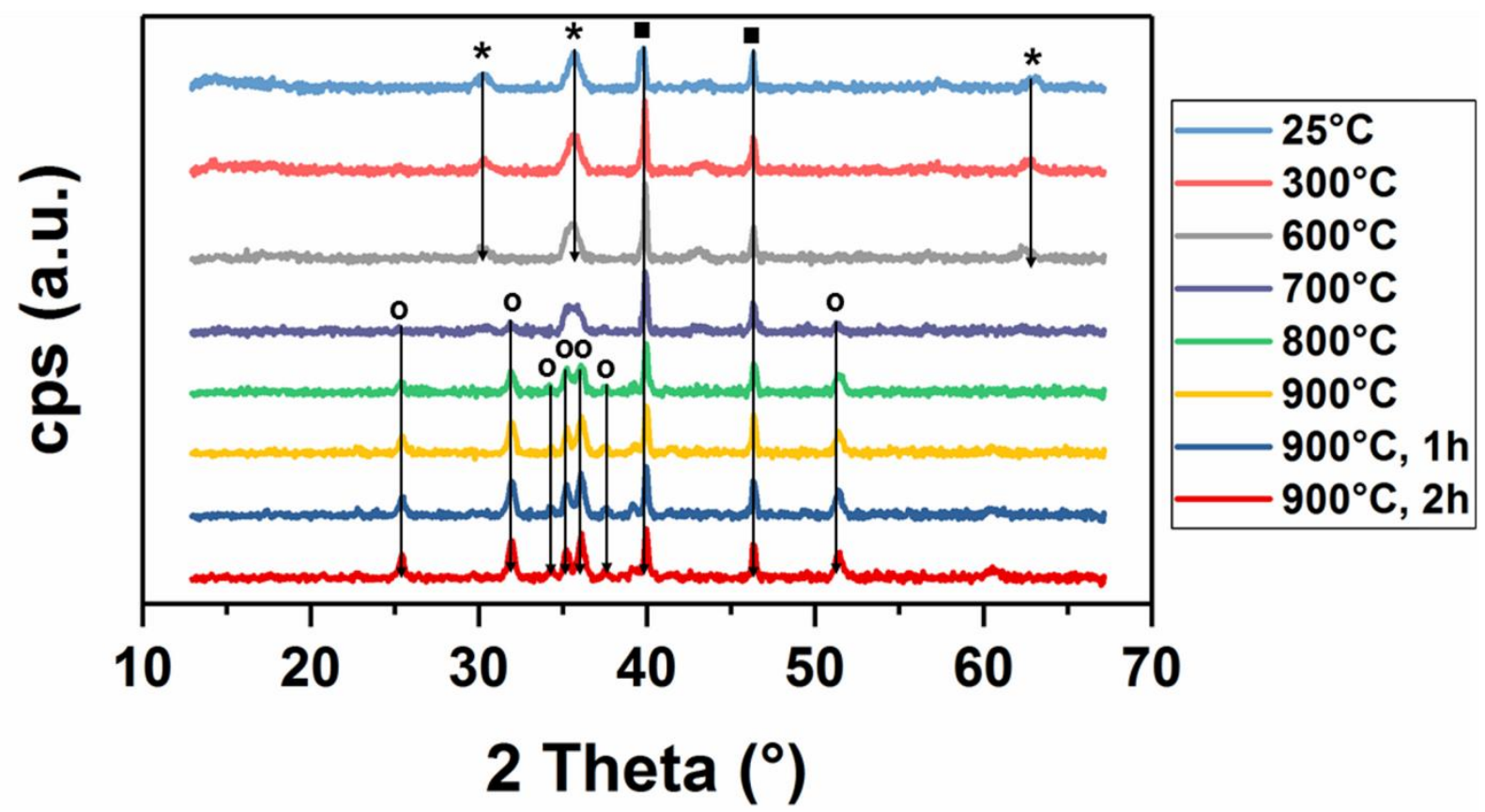

Figure 3. XRD patterns of $\mathrm{Fe}_{3} \mathrm{O}_{4} @ \mathrm{SiO}_{2}$ @ furan resin particles submitted to pyrolysis at different temperatures: Magnetite / maghemite is indicated with "*", fayalite is indicated with "o" and platinum, originating from the employed heating plate, is marked with "ø".

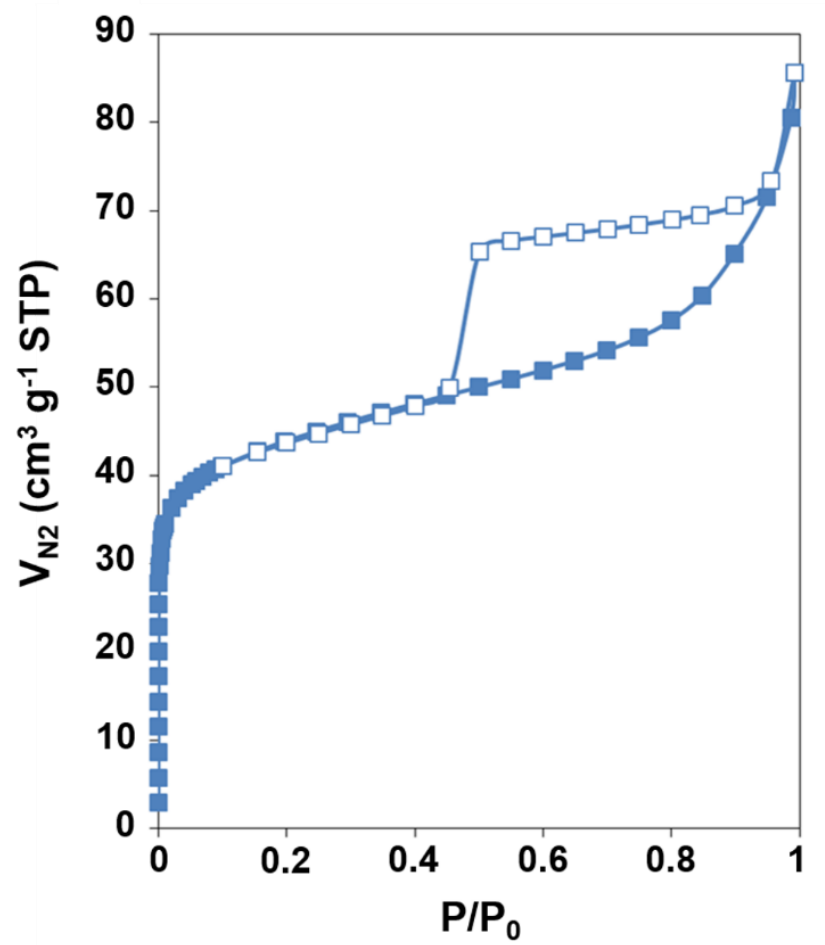

Figure 4. Nitrogen adsorption-desorption at $-196^{\circ} \mathrm{C}$. 
a)

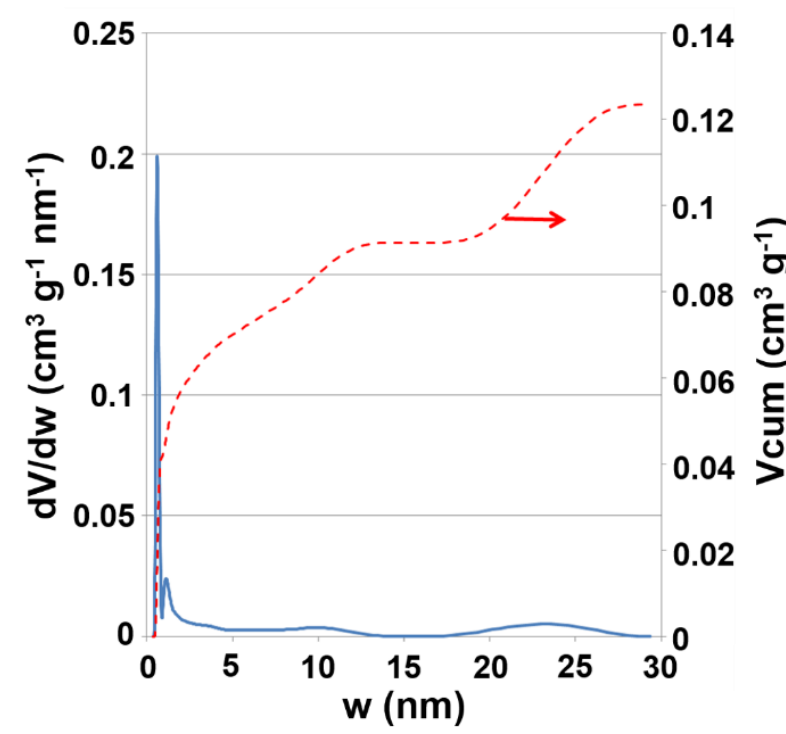

b)

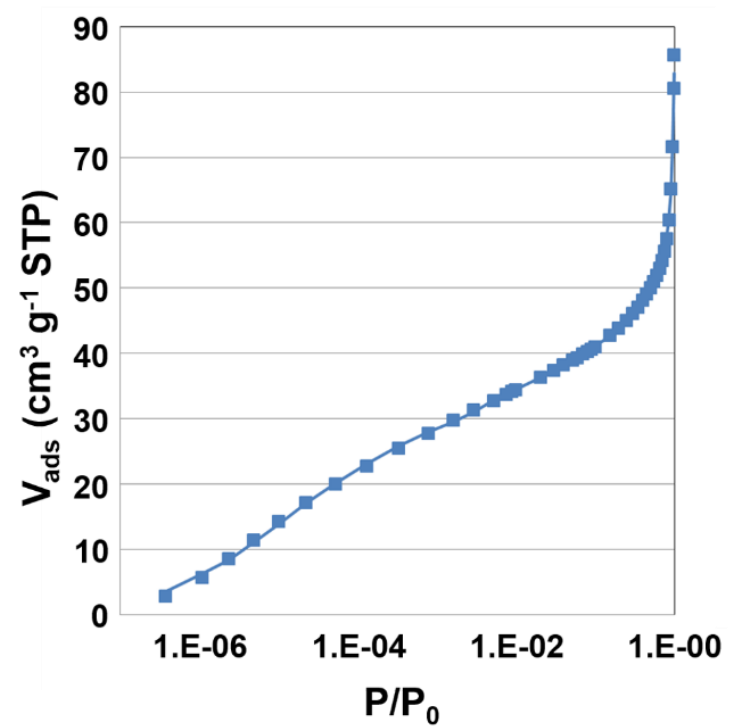

Figure 5. a) Pore size distribution (continuous line) and cumulative pore volume (discontinuous line), determined by application of the 2D-NLDFT HS model, as a function of the pore diameter; b) experimental nitrogen adsorption data (full symbols) fitted with the 2DNLDFT HS model (continuous line)
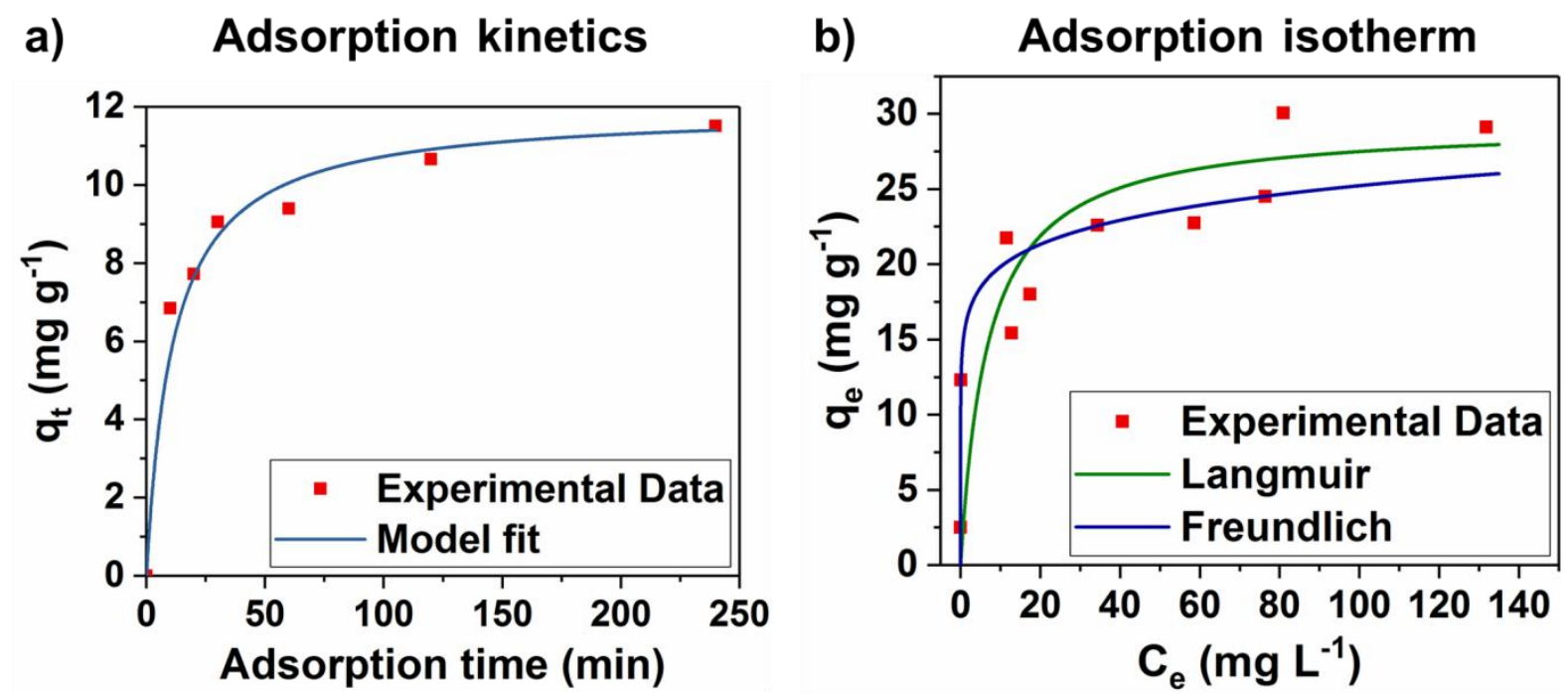

Figure 6. a) Adsorption kinetics of methylene blue on $4 \mathrm{~g} \mathrm{~L}^{-1}$ of carbon-coated particles (Fe$\left.\mathrm{X} @ \mathrm{SiO}_{2} @ \mathrm{C}\right)$ at $22{ }^{\circ} \mathrm{C}$. Initial methylene blue concentration $=100 \mathrm{mg} \mathrm{L}^{-1}, q_{t}=$ amount of adsorbed dye in mg per gram of carbon-coated particles. A pseudo-second-order model is fitted to the experimental data set. b) Adsorption isotherm of methylene blue on carboncoated particles at $22{ }^{\circ} \mathrm{C}$ fitted with Langmuir and Freundlich models. $q_{e}=$ amount of adsorbed dye in mg per gram of carbon-coated particles at equilibrium; $C_{e}=$ concentration of dye in the solution at equilibrium. 

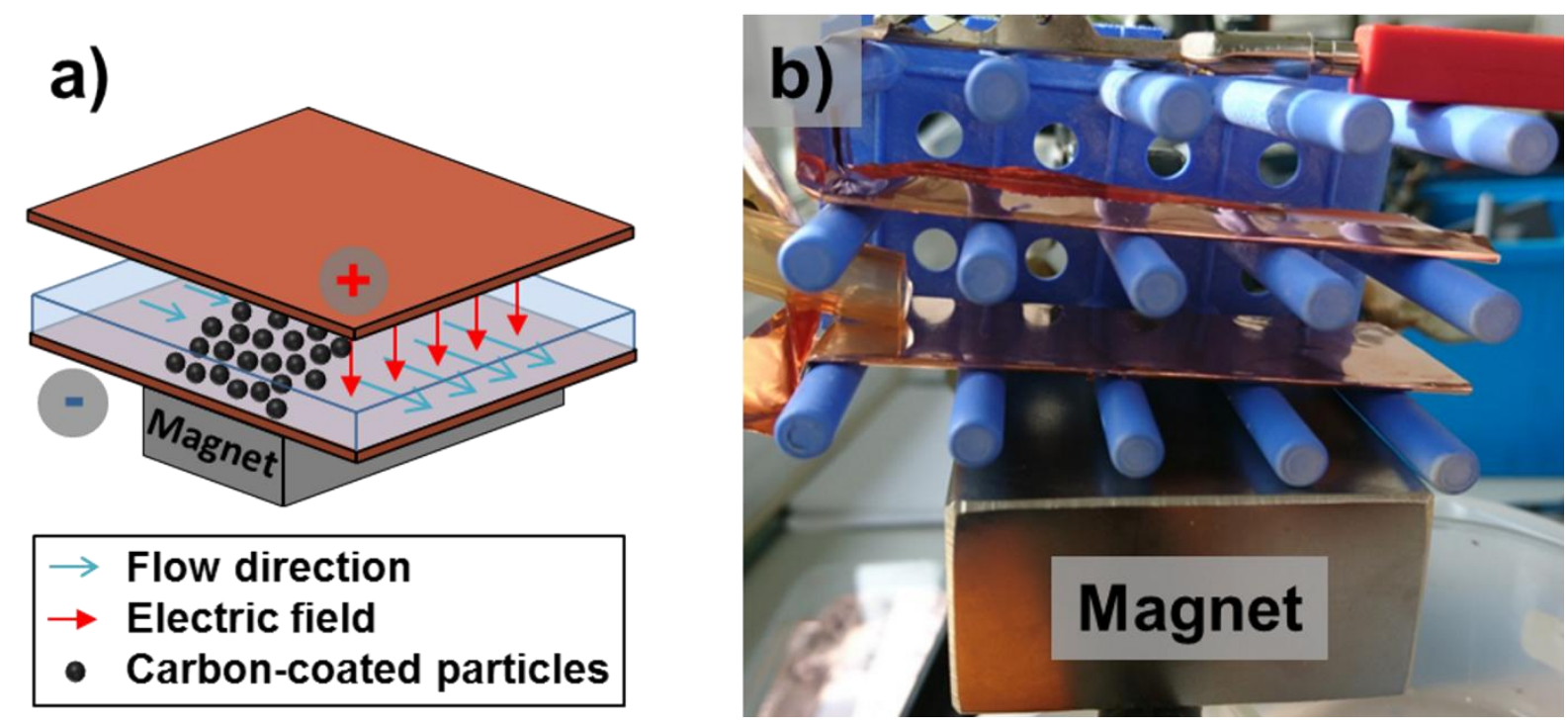

Figure 7. Principle sketch (a) and photograph (b) of the experimental setup for the electrochemical regeneration of the dye-loaded carbon-coated particles $\left(\mathrm{Fe}-\mathrm{X} @ \mathrm{SiO}_{2} @ \mathrm{C}\right)$.

a)

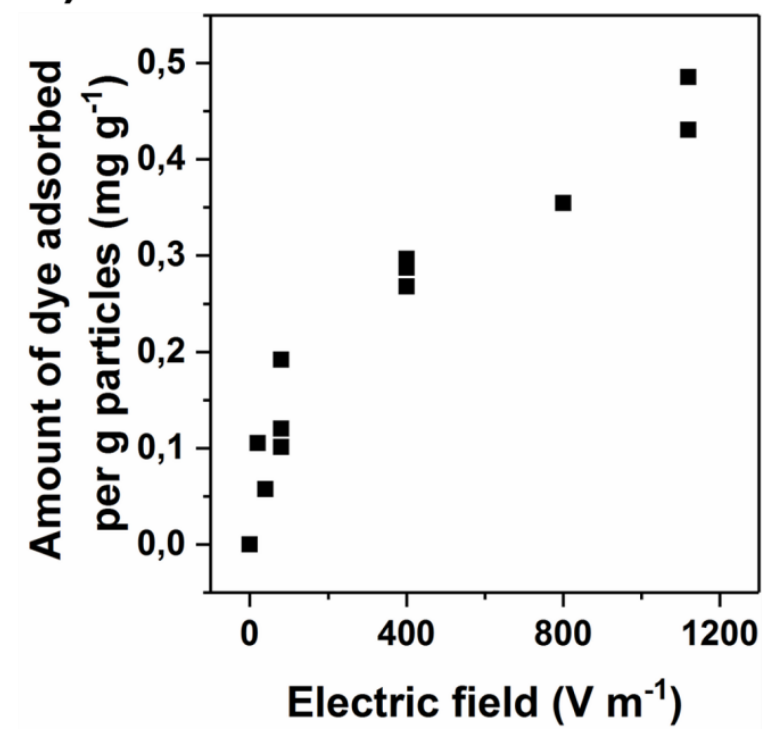

b)

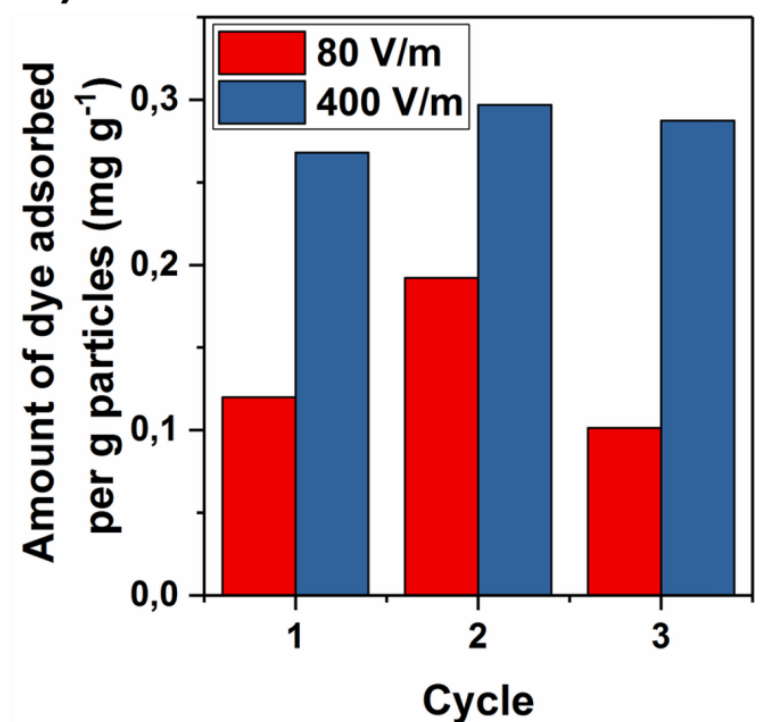

Figure 8. a) Amount of dye adsorbed on the carbon-coated particles after having been fully loaded with dye and subsequently treated in an electric field for regenerating adsorption capacity in relation to the applied electric field. b) Corresponding amount of dye adsorbed on the carbon-coated particles after each regeneration step for three consecutive adsorptionregeneration-cycles while applying an electric potential of either $80 \mathrm{~V} \mathrm{~m}^{-1}$ or $400 \mathrm{~V} \mathrm{~m}^{-1}$, using the same particles in all three cycles.

Table 1. Textural parameters obtained from nitrogen adsorption data at $-196^{\circ} \mathrm{C}$.

\begin{tabular}{ccccccc}
\hline $\begin{array}{c}\mathrm{A}_{\mathrm{BET}} \\
\left(\mathrm{m}^{2} / \mathrm{g}\right)\end{array}$ & $\begin{array}{c}\mathrm{S}_{\mathrm{DFT}} \\
\left(\mathrm{m}^{2} / \mathrm{g}\right)\end{array}$ & $\begin{array}{c}V_{u \mu} \\
\left(\mathrm{cm}^{3} / \mathrm{g}\right)\end{array}$ & $\begin{array}{c}V_{s \mu} \\
\left(\mathrm{cm}^{3} / \mathrm{g}\right)\end{array}$ & $\begin{array}{c}V_{\mu}=V_{u \mu}+V_{\text {s }} \\
\left(\mathrm{cm}^{3} / \mathrm{g}\right)\end{array}$ & $V_{\text {meso }}\left(\mathrm{cm}^{3} / \mathrm{g}\right)$ & $V_{\text {tot }}\left(\mathrm{cm}^{3} / \mathrm{g}\right)$ \\
\hline 164 & 176 & 0.036 & 0.021 & $\begin{array}{c}0.057 \\
(46 \%)\end{array}$ & $0.066(54 \%)$ & 0.123 \\
\hline
\end{tabular}


Table 2. Langmuir and Freundlich equation parameters of methylene blue adsorption onto the carbon-coated particles at $22{ }^{\circ} \mathrm{C}$. Maximum (monolayer) adsorption capacity $q_{\max }$ refers to $\mathrm{mg}$ of methylene blue per $\mathrm{g}$ of carbon-coated particles.

\begin{tabular}{cccccc}
\hline & Langmuir & \multicolumn{3}{c}{ Freundlich } \\
$q_{\max }(\mathrm{mg} / \mathrm{g})$ & $K_{L}(\mathrm{~L} / \mathrm{mg})$ & $R^{2}$ & $n$ & $K_{F}\left((\mathrm{mg} / \mathrm{g})(\mathrm{mg} / \mathrm{L})^{\mathrm{n}}\right)$ & $R^{2}$ \\
\hline 29.4 & 0.146 & 0.975 & 0.104 & 15.6 & 0.750 \\
\hline
\end{tabular}




\section{Table of Content}

A magnetic adsorbent composite particle system was synthesized by coating magnetic $\mathrm{Fe}_{3} \mathrm{O}_{4}$ @ $\mathrm{SiO}_{2}$ microparticles with furan resin and subsequent pyrolysis. Adsorption and regeneration behaviors of the resulting particle system on the model dye methylene blue were tested by use of electrochemical principles.

Keyword: magnetic carbon adsorbents

Michael Schneider, Thomas Ballweg, Lennart Groß, Carsten Gellermann, Angela SánchezSánchez, Vanessa Fierro, Alain Celzard, Karl Mandel**

Magnetic Carbon Composite Particles for Dye Adsorption from Water and their Electrochemical Regeneration

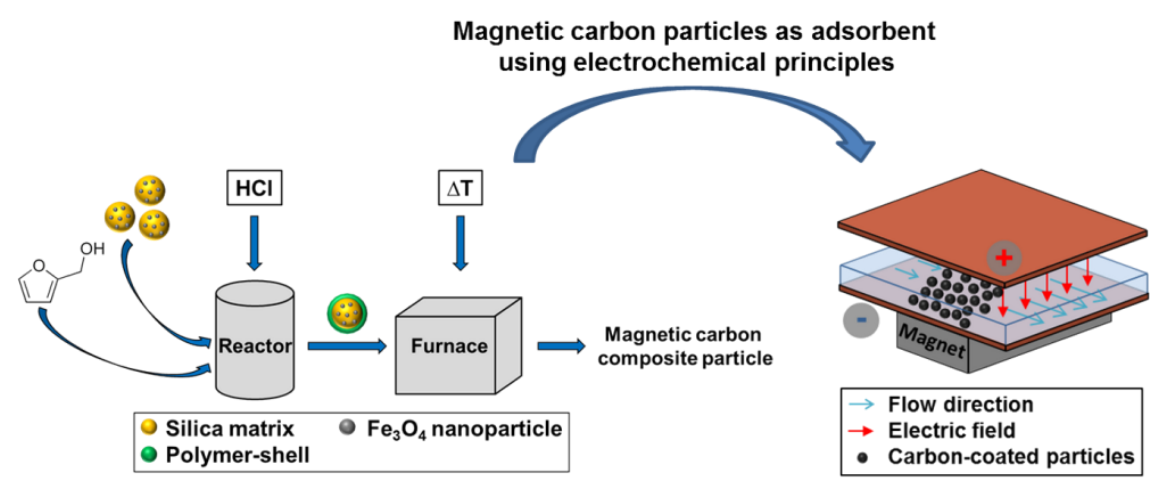

